\title{
Does hyperoxia affect glucose regulation and transport in the newborn?
}

Karim S. Bandali, BSc, MSc ${ }^{a}$
Michael P. Belanger, ALAT
Carin Wittnich, MSc, DVM
a,b,c

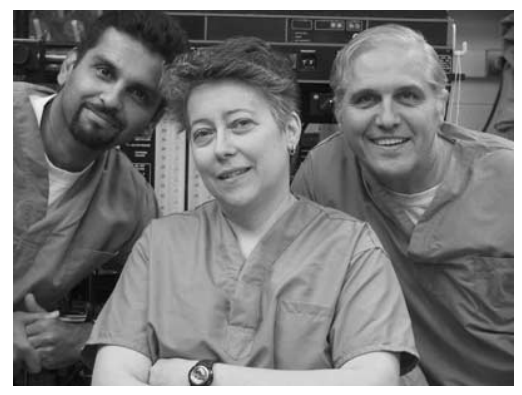

Bandali, Wittnich, Belanger (left to right)
From the Departments of Physiology and Surgery, ${ }^{\mathrm{b}}$ University of Toronto, and The Hospital for Sick Children, ${ }^{\mathrm{c}}$ Toronto, Ontario, Canada.

Supported by The Heart \& Stroke Foundation of Ontario (grant \#T4926). Karim S. Bandali is supported by a doctoral research award from the Heart \& Stroke Foundation of Canada.

Received for publication Feb 4, 2003; revisions requested March 24, 2002; revisions received April 30, 2002; accepted for publication June 18, 2003.

Address for reprints: Dr C. Wittnich, University of Toronto, Medical Sciences Building, Rm 7256, 1 King's College Circle, Toronto, Ontario, Canada M5S 1A8 (Email: c.wittnich@utoronto.ca).

J Thorac Cardiovasc Surg 2003;126:1730-5

Copyright $\odot 2003$ by The American Association for Thoracic Surgery

$0022-5223 / 2003 \$ 30.00+0$

doi:10.1016/S0022-5223(03)01044-4
Objective: Hyperglycemia has been found to occur in children placed on cardiopulmonary bypass. Our laboratory demonstrated that hyperoxia plays a role in this hyperglycemic response and also occurs in the absence of cardiopulmonary bypass. The purpose of this study was to elucidate potential mechanisms underlying the hyperoxic-induced hyperglycemia by examining glucagon, insulin, and epinephrine, which are important in glucose regulation and skeletal and cardiac glucose transporters (GLUT1 and GLUT4), which facilitate glucose entry.

Methods: Three-day-old piglets were anesthetized, intubated, and ventilated to normoxia. Animals were then randomly allocated to either 5 hours of normoxia ( $\mathrm{n}$ $=4)$ or hyperoxia $(n=6)$. Measurements of oxygen, blood glucose, plasma glucagon, insulin, and epinephrine levels were made. Total GLUT1 and GLUT4 content in cardiac and skeletal muscle was measured using Western blotting analysis.

Results: A sustained hyperglycemic response $(P<.001)$ was seen throughout the 5-hour ventilatory period. A significant twofold elevation in glucagon levels $(P<$ $.001)$ and a threefold elevation $(P<.003)$ in plasma insulin levels occurred, despite no significant changes in plasma epinephrine. Total GLUT1 and GLUT4 content were significantly reduced in skeletal muscle by $66 \%$ and $59 \%$, respectively, while no significant changes occurred in cardiac muscle.

Conclusion: This study demonstrates that significant elevations in glucagon and insulin and reductions in total skeletal muscle GLUT1 and GLUT4 content all contribute to hyperoxia-induced hyperglycemia seen in newborns. To optimize postoperative recovery of newborns, consideration should be given to the levels of oxygen used to avoid the potential development of insulin resistance and subsequent decrease in glucose entry.

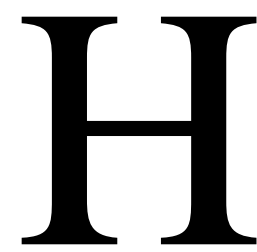

yperglycemia has been found to occur in children suffering from congenital heart disease who are placed on cardiopulmonary bypass (CPB) for primary cardiac repair. ${ }^{1-3}$ Persistent hyperglycemia in children has been shown to cause osmotic diuresis, dehydration, glycosylation of proteins, and an increased incidence in cerebral hemorrhage. ${ }^{4,5}$ Previous studies, mainly performed in adults, have identified several different factors associated with the development of this hyperglycemic response including hypothermia, hypokalemia, and hypocalcemia. ${ }^{6-8}$ However, none of these factors have been consistently implicated. ${ }^{6,9-11}$ Infants and children suffering from congenital heart disease are often exposed to hyperoxia for varying duration during CPB or extracorporeal membrane oxygenation. ${ }^{12-15}$ Under these conditions, systemic hyperoxia can reach an arterial partial pressure of oxygen $\left(\mathrm{PaO}_{2}\right)$ range of 250 to $500 \mathrm{~mm} \mathrm{Hg}$, which can last for 2 to 5 
hours during a cardiac operation or several days during extracorporeal membrane oxygenation. ${ }^{12,15}$

We examined a cohort of children undergoing routine CPB for primary repair for congenital defects at the Hospital for Sick Children (Toronto, Canada) and found that despite normal preoperative blood glucose levels, during surgery they demonstrated a significant and strong positive correlation between their absolute oxygen and blood glucose levels. ${ }^{3}$ This identified that hyperoxia is indeed an important contributor to the hyperglycemic response seen in children who are placed on CPB. This clinical finding was further substantiated by our laboratory using a newborn pig model, which documented that high oxygen levels (hyperoxia) trigger the same hyperglycemic response whether a CPB model was used or just simply a ventilatory model. ${ }^{3}$ This work suggests that hyperoxia may not only play a crucial role in the hyperglycemic response seen during neonatal cardiopulmonary bypass but also that its effect on glucose homeostasis should be considered whenever children are exposed to hyperoxia.

The goal of this study was to begin to elucidate the mechanisms underlying hyperoxia-induced hyperglycemia found in newborns. Due to the rapid nature of this systemic hyperglycemia, the potential role of hormones important in glucose homeostasis including insulin, glucagon, and epinephrine were examined. In addition, as muscle comprises a large portion of body mass (primarily skeletal muscle) and uses glucose as one of its metabolic substrates, any alterations to skeletal and cardiac glucose entry could also have a significant impact on systemic glycemia. Glucose entry in skeletal and cardiac muscle cells is facilitated by glucose transporters: GLUT1 (insulin-independent) and GLUT4 (insulin-dependent). ${ }^{16}$ Work examining the effect of hyperoxia on L6 muscle cell cultures has shown significant decreases in GLUT1 content while reporting no significant changes in GLUT4. In contrast to adult cardiac and skeletal muscle, the fetus has a very high GLUT1 content while the GLUT4 content is reported to be very low. ${ }^{17}$ Because the neonate represents a transition from fetal to adult, both GLUT1 and GLUT4 may be particularly important in facilitating glucose entry in both newborn cardiac and skeletal muscle. The effect of hyperoxia on insulin, glucagon, and epinephrine levels as well as glucose transporter content in newborn heart and skeletal muscle is unknown and was the focus of this study. The ventilatory model used in this study provides an opportunity to begin investigating the underlying mechanisms of hyperoxia-mediated hyperglycemia in the absence of any confounding variables that may be introduced during $\mathrm{CPB}$.

\section{Materials and Methods}

Neonatal Yorkshire pigs were chosen as the animal model in which to study the effects of hyperoxia because the cardiac and pulmonary systems of newborn humans and pigs have many structural and functional similarities. ${ }^{18}$ Yorkshire pigs ( 3 days old, 1.5 to $2.5 \mathrm{~kg}$ ) were used in this study and were provided with sow's milk replacer (Grober Animal Nutrition, Cambridge, Ontario, Canada) until the time of surgery. Animals were anesthetized with an intraperitoneal injection of sodium pentobarbital $(65 \mathrm{mg} /$ kg) (MTC Pharmaceuticals, Cambridge, Ontario, Canada), intubated, and mechanically ventilated to normal blood gases $\left(\mathrm{PaO}_{2}=\right.$ $88 \pm 6 \mathrm{~mm} \mathrm{Hg}, \mathrm{PaCO}_{2}=38 \pm 5 \mathrm{~mm} \mathrm{Hg}$ ) with medical air. Anesthesia was maintained with diluted (1:5) sodium pentobarbital via a catheter in the right carotid artery as needed to maintain an appropriate plane of anesthesia. Normothermia $\left(37.9 \pm 0.2^{\circ} \mathrm{C}\right)$ was maintained in each piglet. A catheter was inserted into the right carotid artery and advanced to the aortic arch to monitor arterial blood pressure via a pressure transducer (COBE, Lakewood, Colo), which was connected to a physiologic recorder (BIOPAC Systems Inc, Goleta, Calif). This catheter was also used for supplemental anesthesia administration, sampling of blood gases, and glucose measurements.

Arterial blood gases $\left(\mathrm{PaO}_{2}\right.$ and $\left.\mathrm{PaCO}_{2}\right)$ and acid-base status $(\mathrm{pH}$ and bicarbonate $\left[\mathrm{HCO}_{3}{ }^{-}\right]$) were monitored at regular intervals using an ABL30 Acid-Base Analyzer (Radiometer, Copenhagen, Denmark), and appropriate ventilatory adjustments ensured that $\mathrm{PaO}_{2}$ and $\mathrm{PaCO}_{2}$ remained at the desired levels.

\section{Experimental Protocol}

Animals were then randomly allocated to 5 hours of normoxia control (84 $\pm 6 \mathrm{~mm} \mathrm{Hg} ; \mathrm{n}=4)$ or hyperoxia $(434 \pm 39 \mathrm{~mm} \mathrm{Hg}$; $\mathrm{n}=6$ ). Measurements of $\mathrm{PaO}_{2}$ were made at hourly intervals. Blood glucose as well as plasma samples for hormone analysis were taken at normoxia baseline, near the halfway point of the ventilatory protocol and at the end of the 5-hour ventilatory protocol.

Blood glucose measurements were made using the Accu-Chek Advantage Blood Glucose Monitoring System (Boehringer Mannheim, Laval, Quebec, Canada). Plasma insulin measurements were made using a porcine insulin enzyme-linked immunosorbent assay (ALPCO Diagnostics, Windham, NH), and plasma glucagon measurements ${ }^{19}$ as well as plasma epinephrine levels were made by radioimmunoassay (ALPCO Diagnostics).

Mixed fiber-type skeletal muscle freeze-clamp biopsy specimens were taken from the hind limb of each piglet at normoxia baseline as well as at the end of the ventilatory protocol, and left ventricular myocardial biopsy specimens were taken only at the end of the ventilatory period for subsequent Western blotting analysis of glucose transporter (GLUT1 and GLUT4).

\section{Total Membrane Isolation}

Skeletal muscle as well as cardiac muscle (left ventricle [LV]) biopsy specimens were homogenized and centrifuged at $1200 \mathrm{~g}$ and $9000 \mathrm{~g}$. Supernatants were then centrifuged at $190,000 \mathrm{~g}$ and the resulting pellet containing total cell membranes was resuspended in a small volume of homogenizing buffer. Protein concentration was assayed by Bradford method using a BCA protein assay kit (Pierce, Rockford, Ill).

\section{Western Blotting Analysis}

Homogenate samples (30 $\mu \mathrm{g}$ of protein) were solubilized in an equal volume of Laemmli sample buffer. Proteins were subjected 


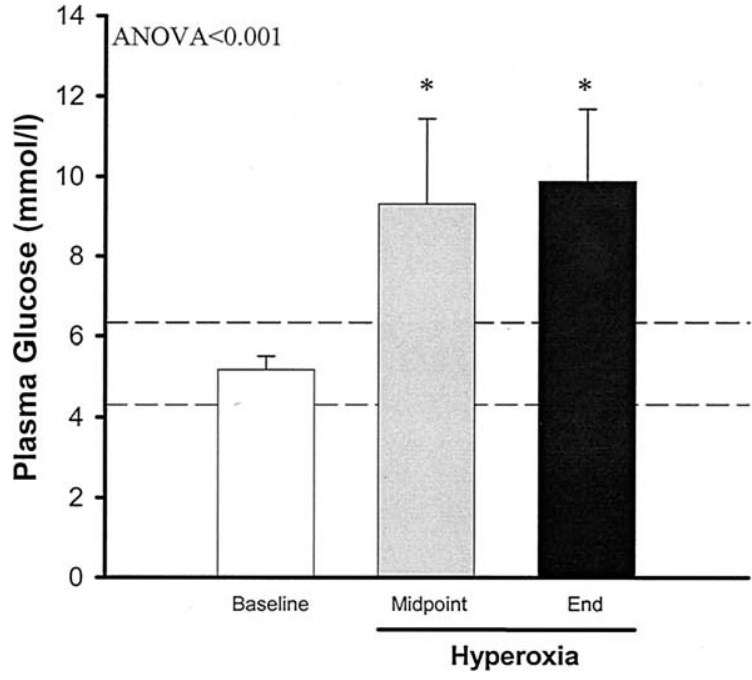

Figure 1. Blood glucose levels taken at baseline (normoxia), near the midpoint, and at the end of $\mathbf{5}$ hours of hyperoxia in newborn pigs. Broken lines represent the range of blood glucose levels measured in normoxic newborn pigs at the same time intervals. ${ }^{*} \boldsymbol{P}<.0001$ versus baseline.

TABLE 1. Hormone measurements at baseline and 5 hours of normoxia

\begin{tabular}{lcc}
\hline Hormone & Baseline value & $\begin{array}{c}5 \text { hours of } \\
\text { normoxia }\end{array}$ \\
\hline Insulin $(\mu \mathrm{g} / \mathrm{mL})$ & $0.096 \pm 0.002$ & $0.103 \pm 0.007$ \\
Glucagon $(\mathrm{pg} / \mathrm{mL})$ & $182.30 \pm 32.54$ & $221.28 \pm 23.33$ \\
Epinephrine $(\mathrm{ng} / \mathrm{mL})$ & $9.58 \pm 0.62$ & $9.53 \pm 1.14$
\end{tabular}

to sodium dodecylsulfate polyacrylamide gel electrophoresis and a Western blotting protocol adapted from Bashan and colleagues. ${ }^{20}$ GLUT protein signals were detected using enhanced chemiluminescence (Amersham Pharmacia Biotech Inc, Piscataway, NJ) and quantified using autoradiography and densitometry.

All experimental procedures and protocols used in this investigation were reviewed and approved by the University of Toronto Animal Care and Use Committee and are in accordance with the National Institute of Health Guide for the Care and Use of Laboratory Animals (NIH publication no. 85-23, revised 1985) and Canadian Council on Animal Care guidelines.

\section{Statistical Analysis}

Repeated measures ANOVA with Duncan multiple range test post hoc was used to identify blood glucose differences between normoxic and hyperoxic groups and across time in the ventilatory model. Paired $t$ tests were used to determine significant differences between baseline and end ventilatory hormone levels in the normoxic group as well as skeletal muscle GLUT1 and GLUT4 content. Student $t$ tests were used to determine differences between LV GLUT1 and GLUT4 content between normoxic and hyperoxic piglets. One-way ANOVA with a Bonferroni post hoc test was

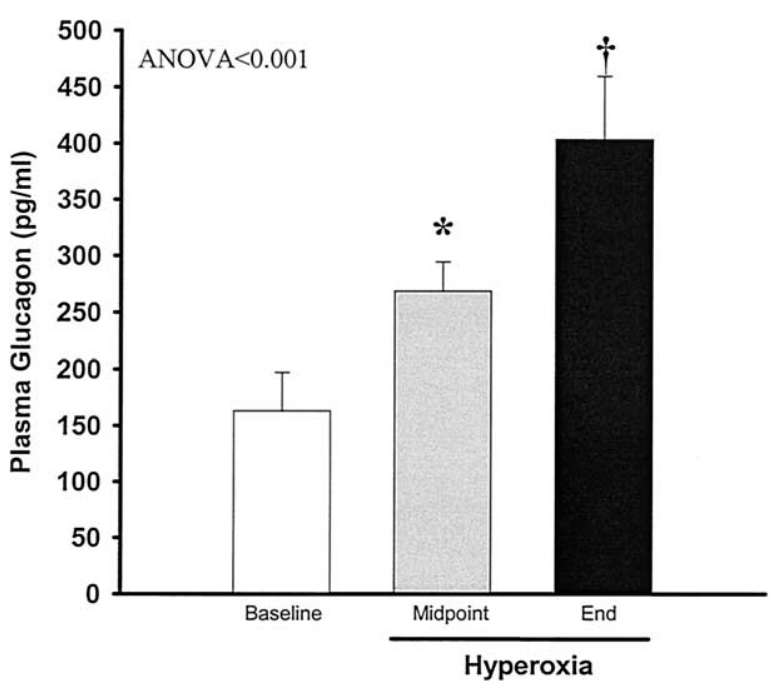

Figure 2. Plasma glucagon levels taken at baseline (normoxia), near the midpoint, and at the end of 5 hours of hyperoxia in newborn pigs. ${ }^{*} P=.007$ versus baseline; ${ }^{\dagger} \boldsymbol{P}=.001$ versus baseline and versus 2.5 hours.

used to analyze difference in hormone levels in the hyperoxic group at baseline and at 2.5- and 5-hour marks, respectively. All data are expressed as mean \pm SEM.

\section{Results}

\section{Development of Hyperglycemia}

This study first confirmed that piglets exposed to hyperoxia had a significant hyperglycemic response compared with piglets exposed to normoxia. This response was well established by the midpoint of the hyperoxic ventilation and did not increase but was sustained over the course of the remainder of the ventilatory period (Figure 1).

\section{Hormones: Plasma Glucagon, Insulin, and Epinephrine}

No significant changes in hormone levels were observed in piglets that underwent 5 hours of normoxic ventilation (Table 1). In contrast, piglets that underwent 5 hours of hyperoxia showed a significant and progressive increase in plasma glucagon (Figure 2) and plasma insulin levels (Figure 3) from baseline. No significant changes were noted in plasma epinephrine levels after 2.5 or 5 hours of hyperoxia (Figure 4). This suggests that both glucagon and insulin play an important role in the development of hyperoxia-induced hyperglycemia.

\section{Glucose Transporters (GLUT1 and GLUT4)}

No significant differences in LV total GLUT1 or GLUT4 content were noted in between normoxic and hyperoxic piglets (Figure 5). In contrast, exposure to 5 hours of hyperoxia resulted in a significant $66 \%$ reduction in skeletal 


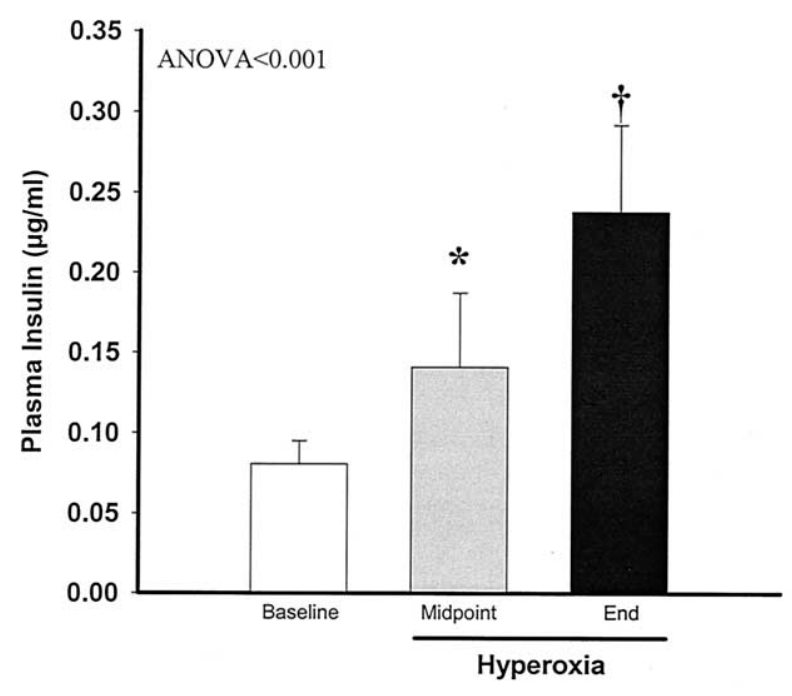

Figure 3. Plasma insulin levels taken at baseline (normoxia), near the midpoint, and at the end of 5 hours of hyperoxia in newborn pigs. ${ }^{*} \boldsymbol{P}=.01$ versus 5 hours; ${ }^{\dagger} \boldsymbol{P}=.001$ versus baseline.

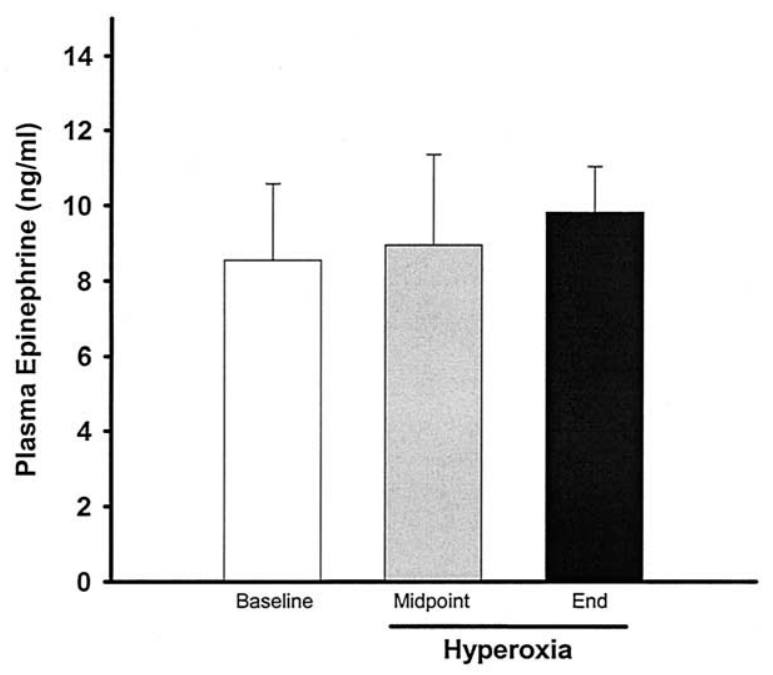

Figure 4. Plasma epinephrine levels taken at baseline (normoxia) near the midpoint and at the end of 5 hours of hyperoxia in newborn pigs.

muscle total GLUT1 content and a 59\% reduction in total GLUT4 content compared with measurements made at baseline normoxia (Figure 6).

\section{Discussion}

Children undergoing surgical repair for congenital heart disease are often placed on CPB during cardiac operations. Hyperoxia is a common occurrence in during CPB and historically the rationale for its use was to overcome the hypothermia-induced shift of the oxygen-hemoglobin dissociation curve and thus ensure adequate oxygen delivery. ${ }^{21}$
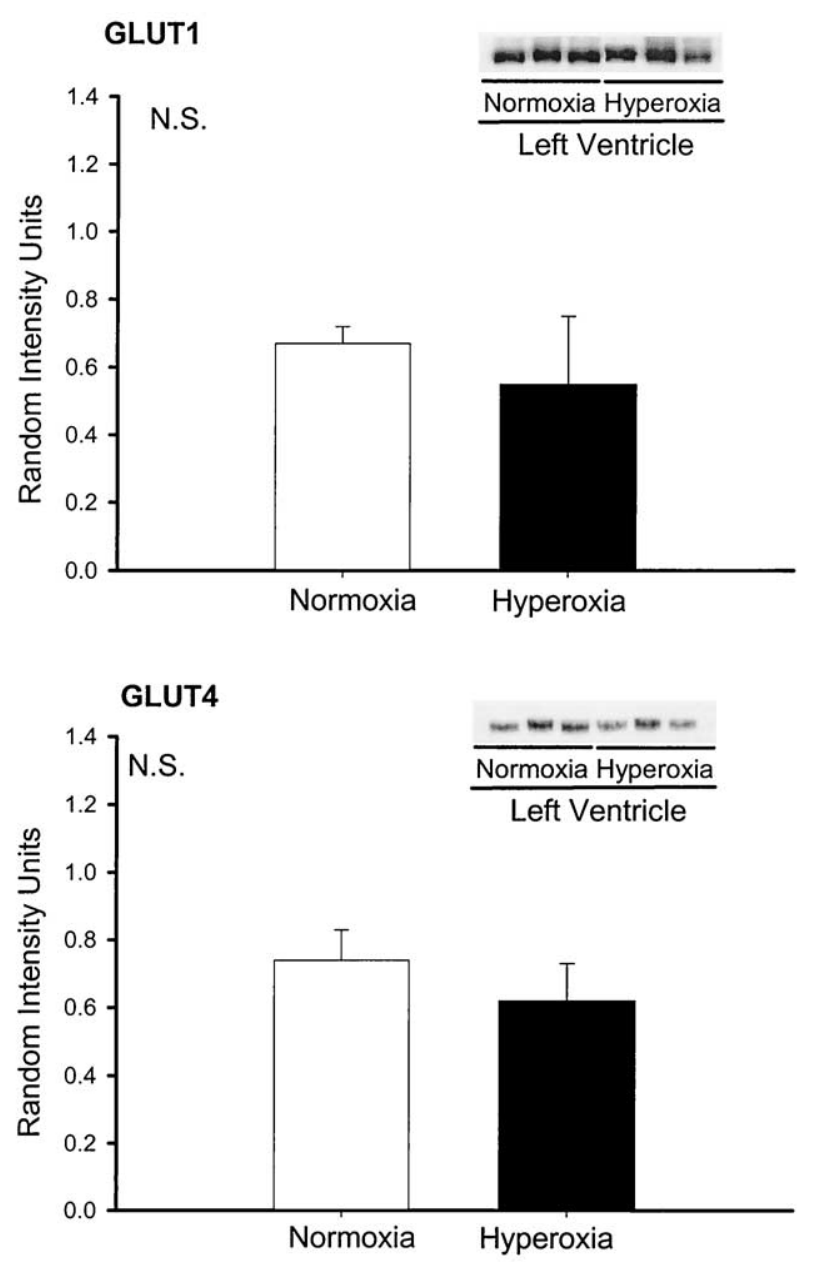

Figure 5. Total LV GLUT1 and GLUT4 content in newborn pigs after 5 hours of normoxia and hyperoxia. Representative Western blots are shown with each graph.

The potentially detrimental effects of hyperoxia are rarely considered. Work in our laboratory has documented that hyperoxia triggers a hyperglycemic response in both ventilatory and CPB settings. Persistent hyperglycemia in children has been shown to cause osmotic diuresis, dehydration, glycosylation of proteins, and an increased incidence in cerebral hemorrhage. ${ }^{4,5}$

It is speculated from the hormone profiles elucidated in this study that the hyperoxia-mediated increase in plasma glucose was driven by significantly elevated levels of glucagon. However, the reason for this significant increase in plasma glucagon levels remains unclear as epinephrine, a known regulator of glucagon release, wasn't shown to significantly change with 5 hours of hyperoxia. However, the direct action of oxygen on the pancreas may be a possible consideration since work by Kobasyashi and colleagues ${ }^{22}$ shows an abundant expression of oxygen-regulated protein (ORP150) in the adult mouse pancreas. They showed that 

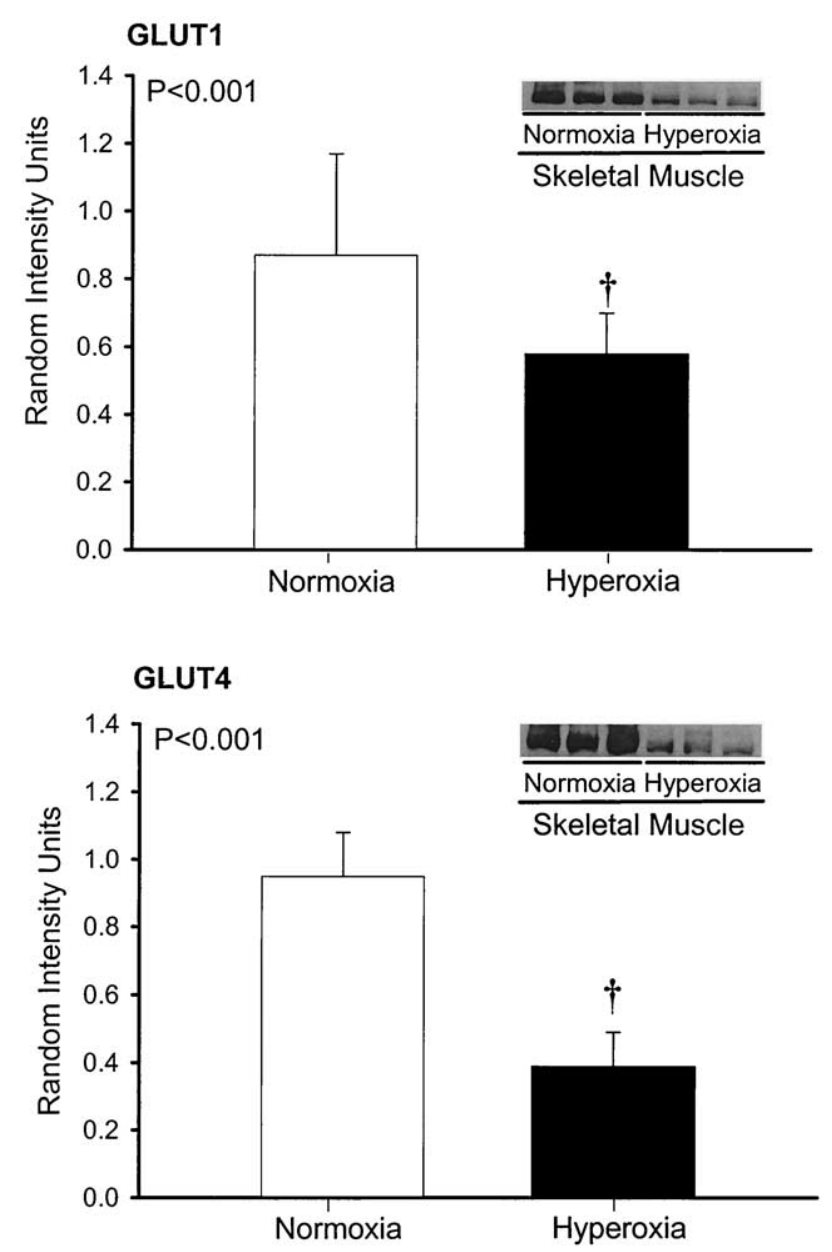

Figure 6. Total skeletal muscle GLUT1 and GLUT4 content in newborn pigs after 5 hours of normoxia and hyperoxia. Representative Western blots are shown with each graph. ${ }^{\dagger} \boldsymbol{P}<.001$ versus normoxia.

when ORP150 expression was increased, glucagon as well as insulin secretion also increased. If hyperoxia causes increases in ORP150 expression then this could account, at least in part, for the increases seen in our study. This is currently unknown, as is whether ORP150 is even present in the newborn.

Along with the marked increase in plasma glucagon, significant increases in plasma insulin levels were also observed, which most likely are a result of the elevated plasma glucose levels. The persistence of hyperglycemia in the face of this marked increase in insulin therefore would imply the potential development of insulin resistance with exposure to hyperoxia. Evidence in the literature does suggest that the development of insulin resistance can be linked with hyperoxia as well as hyperglycemia through the production of oxygen free radicals. Both of these have been shown to increase the production of oxygen free radicals, which can overwhelm the cell's antioxidant systems. ${ }^{23-25}$
Oxygen free radicals have been shown in both adipocyte and muscle cell cultures to be associated with the development of insulin resistance through the inhibition of insulin signaling responsible for the translocation of GLUT4 from intracellular vesicles to the plasma membrane. ${ }^{26-28}$ Some recent preliminary work appears to show that hyperoxia exposure does significantly increase oxygen free radical production, since malondialdehyde levels, which serve as a measure of lipid peroxidation secondary to oxygen free radical-mediated membrane injury in cardiac tissue, went up. Therefore, both hyperoxia and hyperglycemia may contribute to the development of insulin resistance seen in this model through the production of oxygen free radicals.

In addition to the significant alterations in plasma glucagon and insulin levels, this study also documents significant reductions in total content of skeletal muscle GLUT1 and GLUT4. This is an important finding as skeletal muscle is the main tissue involved in the insulin-induced stimulation of glucose uptake and therefore the reduction of its total glucose transporter content represents a major contributor to the hyperoxia-induced hyperglycemia response. The significant reductions in skeletal muscle GLUT1 and GLUT4 may involve both hyperoxia and hyperglycemia documented in this study. However, unlike the mechanism proposed for the development of insulin resistance where both parameters are linked to oxygen free radical production, these 2 parameters may regulate glucose transporters independently in an isoform-specific manner. The findings in this current study are consistent, in part, with L6 muscle cell culture work performed in which 48 hours of hyperoxia led to significant reductions in total GLUT1 content. Therefore, the primary effect of hyperoxia on total glucose transporters content seems to involve the down-regulation of GLUT1. It is unclear whether this regulatory decrease in total GLUT1 content is a result of increased GLUT1 protein degradation or alternatively a result of a decrease in GLUT1 gene transcription.

With respect to GLUT4, this current study documents a significant reduction in total GLUT4 content in skeletal muscle, which is inconsistent with muscle cell culture work that showed no significant change in total GLUT4 content. This discrepancy may be explained in part by the utilization of a whole animal model in this study, which allowed for the presence of other variables affected by hyperoxia such as elevations in glucose and insulin. GLUT4 is known to be regulated by both of these variables. Specifically, GLUT4 has been shown to be internalized, thereby decreasing plasma membrane content in response to hyperglycemia in skeletal muscle, ${ }^{29}$ while work performed in 3T3-L1 mouse adipocytes has shown that in the presence of hyperinsulinemia, there is a down-regulation of GLUT4 mRNA as a result of both rapid repression of transcription of the 
GLUT4 gene and an increased rate of turnover of the GLUT4 message. ${ }^{30}$ If similar mechanisms are at work in the newborn model used in this study where hyperinsulinemia has been clearly documented to accompany the hyperglycemic response, then this may provide a potential explanation for the significant reduction in total GLUT4 content. Therefore, the regulation of GLUT1 and GLUT4 expression in skeletal muscle may involve both oxygen and metabolism.

In contrast to the significant reductions in total glucose transporter content seen in skeletal muscle, myocardial GLUT1 and GLUT4 showed a smaller reduction in total content that did not reach statistical significance. Even though reductions in total glucose transporter content were surprisingly not observed in the heart, the potential development of insulin resistance seen in this study may still limit glucose entry and compromise newborn heart function. Because the contribution of skeletal muscle to insulin-induced stimulation of glucose uptake is greater compared with the newborn heart, the systemic hyperglycemia seen in this study can still be attributed in part to the reductions in total GLUT1 and GLUT4 content in skeletal muscle.

In conclusion, this study demonstrated that significant elevations in glucagon and insulin and reductions in total skeletal muscle GLUT1 and GLUT4 content all contribute to hyperoxia-induced hyperglycemia seen in newborns. As a result of these changes, these findings highlight the systemic nature of the effects of hyperoxia, which may have potentially important implications concerning the overall postoperative recovery of newborns placed on $\mathrm{CPB}$ for primary cardiac repair. Therefore, to optimize postoperative recovery of newborns, consideration may need to be given to adjustments in levels of oxygen used clinically to avoid the potential development of insulin resistance and subsequent decrease in glucose entry.

We wish to thank Dr P. Brubaker for her assistance with the glucagon radioimmunoassay.

\section{References}

1. Barratt-Boyes BG, Neutze JM, Seelye ER, Simpson M. Complete correction of cardiovascular malformations in the first year of life. Prog Cardiovasc Dis. 1972;15:229-53.

2. Pooley RW, Hayes CJ, Edie RN, Gersony WM, Bowman FO, Malm JR. Open-heart experience in infants using normothermia and deep hypothermia. Ann Thorac Surg. 1976;22:415-23.

3. Bandali KS, Belanger MP, Wittnich C. Is hyperglycemia seen in neonates during cardiopulmonary bypass due to hyperoxia? $J$ Thorac Cardiovasc Surg. 2001;122:753-8.

4. Plides RS. Neonatal hyperglycemia. J Pediatr. 1986;109:905-7.

5. Dweck HS, Cassady G. Glucose intolerance in infants of very low birth weights: incidence of hyperglycemia in birth weights $1100 \mathrm{gms}$ or less. Pediatrics. 1974;53:189-95.

6. Brown TCK, Dunlop ME, Stevens BJ, Clarke CP, Shanahan FA.
Biochemical changes during surface cooling for deep hypothermia in open-heart surgery. J Thorac Cardiovasc Surg. 1972;65:402-8.

7. Howell SL, Taylor KW. Potassium ions and secretions of insulin by islets of Langerhans incubated in vitro. Biochem J. 1968;108:17-24.

8. Curry DL, Curry KP. Hypothermia and insulin secretion. Endocrinology. 1970;87:750-5.

9. Benzing G, Francis PD, Kaplan S, Helmsworth JA, Sperling MA. Glucose and insulin changes in infants and children undergoing hypothermic open-heart surgery. Am J Cardiol. 1983;52:133-6.

10. Mandelbaum I, Morgan CR. Effect of extracorporeal circulation upon insulin. J Thorac Cardiovasc Surg. 1968;55:526-34.

11. Moffitt EA, Roseavear JW, Molnar GD, McGoon DC. Myocardial metabolism in open-heart surgery. Correlation with insulin response. J Thorac Cardiovasc Surg. 1970;59:691-706.

12. Allen BS, Barth MJ, Ilbawi MN. Pediatric myocardial protection: an overview. Semin Thorac Cardiovasc Surg. 2001;13:56-72.

13. Wittnich C. Pediatric cardiopulmonary bypass and heart preservation. Can J Pediatr. 1991;3:6-13.

14. Rosenberg EM, Cook LN. Electromechanical dissociation in newborns treated with extracorporeal membrane oxygenation: an extreme form of cardiac stun syndrome. Crit Care Med. 1991;19:780-4.

15. Aoshima M, Yokota M, Shiraishi Y. Prolonged aortic cross clamping in early infancy and method of myocardial preservation. J Cardiovasc Surg (Torino). 1988;29:591-5.

16. Gould GW, Holman GD. The glucose transporter family: structure, function and tissue-specific expression. Biochem J. 1993;255:329-41.

17. Santalucia T, Camps M, Castello A, et al. Developmental regulation of GLUT-1 (erythroid/Hep G2) and GLUT4 (muscle/fat) glucose transporter expression in rat heart, skeletal muscle and brown tissue. Endocrinology. 1992;130:837-45.

18. Glauser EM. Advantages of piglets as experimental animals in pediatric research. Exp Med Surg. 1966;24:181-90.

19. Dhanvantari S, Seidah NG, Brubaker PL. Role of prohormone convertases in the tissue-specific processing of proglucagon. Mol Endocrinol. 1996;10:342-55.

20. Bashan N, Burdett E, Hundal HS, Klip A. Regulation of glucose transporter and GLUT1 glucose transporter expression by $\mathrm{O}_{2}$ in muscle cell cultures. Am J Physiol. 1992;262:C682-90.

21. Wittnich C, Torrance SM, Carlyle CE. Effects of hyperoxia on neonatal myocardial energy status and responses to global ischemia. Ann Thorac Surg. 2000;70:2125-31.

22. Kobayashi T, Ogawa S, Yura T, Yanagi H. Abundant expression of $150-\mathrm{kDa}$ oxygen-regulated protein in mouse pancreatic beta cells is correlated with insulin. Biochem Biophys Res Comm. 2000;267:831-7.

23. Jamieson D, Chance B, Cadenas E, Boveris A. The relation of free radical production to hyperoxia. Аппи Rev Physiol. 1986;48:703-19.

24. Ceriello A. Acute hyperglycemia and oxidative stress generation. Diabet Med. 1997;14:S45-9.

25. Ceriello A. Oxidative stress and glycemic regulation. Metabolism. 2000;49(Suppl 1):27-9.

26. Rudich A, Tirosh A, Potashnick R, Hemi R, Kanety H, Bashan N. Prolonged oxidative stress impairs insulin-induced GLUT4 translocation in 3T3-L1 adipocytes. Diabetes. 1998;47:1562-9.

27. Hansen LL, Ikeda Y, Olsen GS, Busch AK, Mosthaf L. Insulin signaling is inhibited by micromolar concentrations of $\mathrm{H}_{2} \mathrm{O}_{2} . J$ Biol Chem. 1999;274:25078-84.

28. Maddux BA, See W, Lawrence JC, Goldfine AL, Gloldfine ID, Evans JL. Protection against oxidative stress-induced insulin resistance in rat L6 muscle cells by micro concentrations of alpha-lipoic acid. Diabetes. 2001;50:404-10.

29. Mathoo JM, Shi ZQ, Klip A, Vranic M. Opposite effects of acute hypoglycemia and acute hyperglycemia on glucose transport and glucose transporters in perfused rat skeletal muscle. Diabetes. 1999;48: 1281-8.

30. Flores-Riveros JR, McLenithan JC, Ezake O, Daniel Lane M. Insulin down-regulated expression of the insulin-responsive glucose transporter (GLUT4) gene: effects on transcription and mRNA turnover. Proc Natl Acad Sci U S A. 1993;90:512-6. 